Supporting information for:

\title{
Ionic Liquid Assisted Alignment of Corn Straw Microcrystalline Cellulose Aerogels with Low Tortuosity Channels for Salt-assistance Solar Steam Evaporators
}

Jiyan Li*, Xu Zhou, Yanju Jing, Hanxue Sun, Zhaoqi Zhu, Weidong Liang and An Li*

College of Petrochemical Technology, Lanzhou University of Technology, Lanzhou 730050, P. R. China

*Corresponding Author. E-mail: lijiyan3@163.com; E-mail: lian2010@1ut.cn. 


\section{Supporting Figures:}

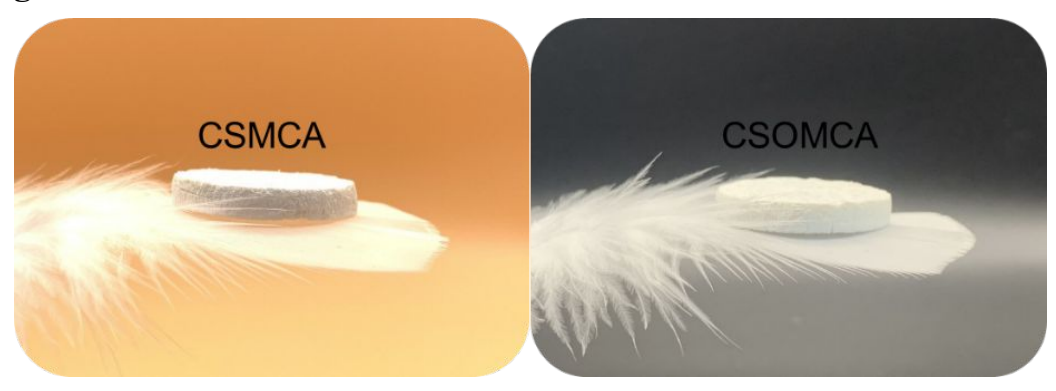

Figure S1. Ultralight aerogels can be placed on feathers.
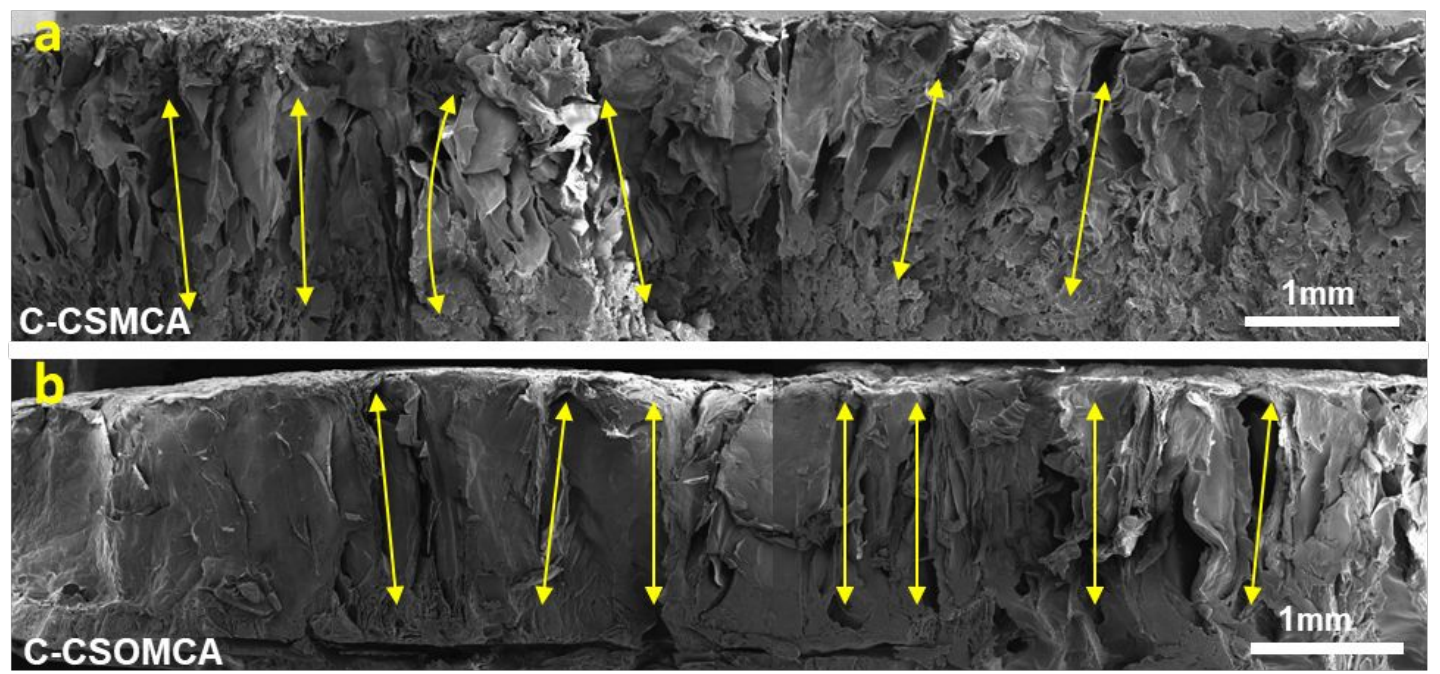

Figure S2. (a) Longitudinal SEM images of C-CSMCA. (b) Longitudinal SEM images of C-CSOMCA.

\section{Supporting Notes:}

\section{Note S1. The proportion of microcrystalline cellulose and oxidized microcrystalline cellulose}

We had fabricated 3 kinds of aerogel based on the different ratios of microcrystalline cellulose and oxidized microcrystalline cellulose. Among them, the aerogel made of pure microcrystalline cellulose had the largest pores and formed a stable three-dimensional net structure. However, the structure of aerogel made from pure oxidized microcrystalline cellulose was relatively fragile and easily damaged during the replacement of ionic liquid. This is because, compared with pure microcrystalline cellulose aerogel, pure microcrystalline oxycellulose aerogel had thinner and shorter fiber structures, and the hydrogel formed during the regeneration process has low binding force, making it difficult to form a stable three-dimensional and the network structure is more likely to collapse. Therefore, on this basis, we prepared microcrystalline oxycellulose aerogel at a ratio of 1:1 (microcrystalline cellulose: oxidized microcrystalline cellulose). 


\section{Note S2. Chemical}

$\mathrm{NaOH}, \mathrm{NaBr}$ and ethanol were purchased from Sinopharm chemical reagent Co., Ltd. $\mathrm{NaClO}_{2}$ was obtained from Tianjin Ding Sheng Xin chemical industry Co., Ltd. Hydrochloric acid was purchased from Baiyin Liangyou Chemical Reagents Co., LTD. TEMPO and isopropanol were supplied by Shanghai Aladdin Bio-Chem Technology Co., LTD. NaClO was obtained from Tianjin Tianhe Chemical Co., Ltd. [BMIm]Cl was purchased from Lanzhou Aolike Chemical Co., Ltd.

\section{Note S3. Description of the testing procedure for the material}

SEM: The microstructures were recorded by using scanning electronic microscope (SEM, JSM-6701F, JEOL, Ltd.). Due to the poor electrical conductivity of cellulose, in addition to preparing the sample sheet as required $\left(1 \times 1 \times 0.5 \mathrm{~cm}^{3}\right)$, it's also necessary to spray gold nanoparticles on sample's surface. Then the sample will be put under the scanning electronic microscope to observe the microstructure of surface.

Mercury intrusion porosimetry (MIP): High prformance automatic mercury compressor (AutoPore IV 9500) was used to test the pore structure of samples. Non-wettable mercury is forced into the pores of the sample under pressure. The different pore size of the sample requires different invasion pressure, and the amount of mercury into the pore size is different. The pore size distribution of the sample is obtained from the data.

Compressive resistance: The tests of samples' compressive resistance were measured on electronic universal teasing machine (CMT-2, China). The regular cylinder sample is placed on the load-bearing surface of the universal testing machine, and the compression surface is close to the sample. Next the specifications of the sample are entered into the computer and set the compression ratio, and then the compression is carried out to get the relationship between the sample deformation ratio and pressure.

Light absorption: UV-Vis spectrometer (PerkinElmer Lambda 750s, US) was used to test the light absorption of samples. First, the barium sulfate was pressed into sheet to test the absolute reflectivity under the light wavelength of $240-2500 \mathrm{~nm}$, and the background was collected. Finally, the sheet sample $\left(1 \times 1 \times 0.5 \mathrm{~cm}^{3}\right)$ was put into the test to get the absolute reflectivity.

FTIR: The fourier transform infrared spectroscopy was recorded by using $\mathrm{KBr}$ particles with a Nexus 670 spectrophotometer. After grinding the potassium bromide and sample (100:1) for 10 
minutes, the mixture was pressed into flakes and then placed into the Nexus 670 spectrophotometer for testing. Under the irradiation of infrared light with continuous frequency changes, the characteristic peak data of chemical functional groups was obtained.

XPS: X-ray photoelectron (PHI 5702, US) was used to test the sample's elemental composition and molecular structure. When the sample powders are irradiated by X ray in the vacuum state, the outer electrons or valence electrons in atoms or molecules are excited to form photoelectrons, and the chemical composition of the sample is determined by measuring its energy and quantity.

Thermal conductivity: Thermal conductivity measuring instrument (Hot Disk TPS2500, Sweden) recorded the thermal conductivity of the sample. The probe is sandwiched between two samples with flat surface, diameter of $2 \mathrm{~cm}$ and height of $0.5 \mathrm{~cm}$. The probe will automatically heat and detect the temperature of the two samples, and the thermal conductivity can be obtained by calculating the relationship between time and sample temperature.

The surface temperature: The IR thermal camera (Thermal Imager TESTO 869, China) recorded the temperature changes of sample surface.

Photothermal conversion test: Solar simulator (Scientech SF300, Canada) equipped with an acrylic Fresnel lens (Greenbrier International, Canada) was the main experimental equipment of steam generation. First, we put the transparent glass vial containing water and samples on a precision electronic balance, and then adjust the light intensity of the solar simulator to irradiate the top layer of the sample floating on the water surface. The relationship between the mass change of the electronic balance and irradiation time are recorded. At the same time, the IR thermal camera focused on the center of the sample surface to record the temperature change.

Ion concentration: Inductively coupled plasma mass spectrometry (Agilent ICP-MS 7700, US) was used to test the ionic concentration.

Methylene blue absorption spectrum: Absorption spectra were measured on a UV-Vis spectrometer (Lambda 900, US). Add the liquid sample to the $3 / 4$ of cuvette, and the sample will absorb the specific wavelength light emitted by the instrument. Then the absorption value of sample is converted into the concentration of the substance contained in the sample. 